Instituto Internacional de Investigación y Desarrollo Tecnológico Educativo INDTEC, C.A.

DOI: https://doi.org/10.29394/Scientific.issn.2542-2987.2021.6.20.2.41-61

OAI-PMH: http://www.indteca.com/ojs/index.php/Revista Scientific/oai

Artículo Original / Original Article

\title{
Síndrome de Burnout en Odontólogos del Seguro Social Campesino de Chimborazo en el año 2020
}

\author{
Autores: Caterine Nataly Orozco Orozco \\ Universidad Regional Autónoma de los Andes, UNIANDES \\ kathi 2815@hotmail.com \\ Riobamba, Ecuador \\ https://orcid.org/0000-0002-0024-2689 \\ Darwin Raúl Noroña Salcedo \\ Universidad Regional Autónoma de los Andes, UNIANDES \\ darwin norona@yahoo.com \\ Quito, Ecuador \\ https://orcid.org/0000-0002-0630-0456 \\ Vladimir Vega Falcón \\ Universidad Regional Autónoma de los Andes, UNIANDES \\ vega.vladimir@gmail.com \\ Ambato, Ecuador \\ https://orcid.org/0000-0003-0140-4018
}

\section{Resumen}

El Síndrome de Burnout es provocado como consecuencia inadecuada al estrés laboral crónico. El objetivo fue determinar el grado de afectación del Síndrome de Burnout en odontólogos del Seguro Social Campesino de Chimborazo en el año 2020. Fue un estudio de tipo observacional, descriptivo, transversal y prospectivo. Se trabajó con toda la población de 43 profesionales. Se utilizó como herramienta el test MBI-GS, que evalúo el Síndrome de Burnout en tres dimensiones y se ha constituido en el Gold estándar, comprendiendo 16 ítems. Como resultados se obtuvo una baja eficacia profesional, dimensión presentada con mayor porcentaje alcanzando $76,7 \%$ (33 personas), desgaste emocional con $44,2 \%$ (19 personas) y finalmente el cinismo con 39,5\% (17 personas). No se registraron inferencias entre los criterios nominales de edad, género y estado civil. Dos personas registraron tres dimensiones en alto, 14 trabajadores con dos, 12 personas con una dimensión. Finalmente, 15 odontólogos no registraron factores con exposición alta. Como conclusión se encontró bajo porcentaje del Síndrome de Burnout. La eficacia profesional mayormente afectada, seguido del cansancio emocional y el cinismo, permitiendo tomar acciones y establecer lineamientos para controlar las dimensiones afectadas y evitar la incidencia del Síndrome de Burnout. profesional.

Palabras clave: síndrome de burnout; estrés; odontología; test MBI-GS, eficacia

Código de clasificación internacional: 3204.03 - Salud profesional.

\section{Cómo citar este artículo:}

Orozco, C., Noroña, D., \& Vega, V. (2021). Síndrome de Burnout en Odontólogos del Seguro Social Campesino de Chimborazo en el año 2020. Revista Scientific, 6(20), 41-61, e-ISSN: 25422987. Recuperado de: https://doi.org/10.29394/Scientific.issn.2542-2987.2021.6.20.2.41-61

Fecha de Recepción: 13-01-2021
Fecha de Aceptación: 25-03-2021
Fecha de Publicación: 05-05-2021 
OAl-PMH: http://www.indteca.com/ojs/index.php/Revista Scientific/oai

\title{
Artículo Original / Original Article
}

\section{Burnout Syndrome in Dentists of the Peasant Social Security of Chimborazo in the year 2020}

\begin{abstract}
Burnout Syndrome is caused as an inappropriate consequence of chronic work stress. The objective was to determine the degree of affectation of the Burnout Syndrome in dentists of the Peasant Social Security of Chimborazo in the year 2020. It was an observational, descriptive, crosssectional and prospective study. We worked with the entire population of 43 professionals. The MBI-GS test was used as a tool, which assesses the Burnout Syndrome in three dimensions and has become the Gold standard, comprising 16 items. As a result, a low professional efficacy was obtained, a dimension presented with the highest percentage reaching $76.7 \%$ (33 people), emotional exhaustion with $44.2 \%$ (19 people) and finally cynicism with $39.5 \%$ (17 people). No inferences were made between the nominal criteria of age, gender and marital status. Two people registered three dimensions high, 14 workers with two, 12 people with one dimension. Finally, 15 dentists did not register factors with high exposure. As a conclusion, a low percentage of Burnout Syndrome was found. The most affected professional efficiency, followed by emotional fatigue and cynicism, allowing to take actions and establish guidelines to control the affected dimensions and avoid the incidence of Burnout Syndrome.
\end{abstract}

Keywords: burnout syndrome; stress; odontology; MBI-GS test, professional efficiency.

International classification code: 3204.03 - Occupational health.

How to cite this article:

Orozco, C., Noroña, D., \& Vega, V. (2021). Burnout Syndrome in Dentists of the Peasant Social

Security of Chimborazo in the year 2020. Revista Scientific, 6(20), 41-61, e-ISSN: 2542-2987.

Recovered from: https://doi.org/10.29394/Scientific.issn.2542-2987.2021.6.20.2.41-61

Date Received:

13-01-2020
Date Acceptance:

25-03-2020
Date Publication: 05-05-2021 


\section{Introducción}

Es necesario recordar a Ogdon y Díaz-Reissner (2017a): quienes interpretan el Síndrome de Burnout como una disfunción psicológica, que aparece como consecuencia del desgaste emocional crónico que se vincula con la actividad laboral excesiva, exigente, que provoca un desequilibrio sistémico, alterando la productividad profesional y las relaciones interpersonales.

En conformidad con Maslach y Jackson (1986), citados por Huayhua (2017): el Burnout o síndrome del quemado, se estudia desde la época de los años 80 , y es descrito por primera vez en el año 1974 por el psiquiatra Herbert Freudenberger (1926-1999), que lo define como una sensación de fatiga o sentimiento de estar frustrado ocasionado por la concesión a una causa, estilo de vida o nexos que no alcanzan los resultados esperados; y en 1981 lo definen como un síndrome caracterizado por despersonalización, baja realización personal y cansancio emocional, que sucede en contextos laborales.

Para la explicación, descripción y análisis del burnout, generalmente se utiliza el modelo de Maslach basado en tres condiciones importantes: la despersonalización, el agotamiento emocional y la ineficacia personal. La experiencia y capacidad de autoadaptibilidad que demuestra una persona frente a situaciones de estrés físico y cansancio mental, toma vital importancia en el trajinar cognitivo, la pérdida progresiva de energía, el desgaste y la poca receptibilidad a las funciones asignadas.

Por otro lado, la despersonalización, atrae un paradigma de frustración, miedo desinterés, irritabilidad, poca tolerancia frente a situaciones de índole profesional, escasa afinidad por el trabajo grupal, actitudes incompatibles con el adecuado desarrollo personal, colectivo y social. Finalmente, la baja realización personal engloba un sentimiento de incapacidad que muestra el individuo para desempeñar su trabajo, a causa de esto, surgen situaciones de 
prácticas poco éticas como impuntualidad e inestabilidad laboral.

Interpretando a Chacaltana y Rojas (2019): la Organización Mundial de la Salud (OMS) reconoce al Burnout como un factor de riesgo laboral, debido a la forma de afectar la calidad de vida y la salud mental, llegando a poner en riesgo la integridad del individuo que lo sufre, el impacto se traduce en un $40 \%$ de incidencia de este síndrome a nivel mundial. En tal virtud, desde que se identifica el síndrome de agotamiento emocional como un elemento interviniente en enfermedades psicosociales, se diseñan múltiples y constantes actividades que permiten la disminución de la probabilidad de su ocurrencia en los distintos centros de trabajo. El contexto es amplio, por tanto, la OMS aboga por investigaciones adicionales centradas en la prevención.

Por otra parte, Meyerson, Gelkopf, Eli y Uziel (2020): indican que, en Ecuador son varios los estudios que muestran la prevalencia del Síndrome de Burnout en el personal de salud, concretamente en médicos, enfermeros, y auxiliares; no obstante, son escasas las investigaciones encaminadas a la detección de este desorden psicoemocional en profesionales odontólogos, quienes se expone a un número mayor de riesgos laborales durante la pandemia COVID-19, ya que tienen que colaborar en actividades de apoyo en procedimientos de salud. Si se suman las condiciones de trabajo y la probabilidad de ocurrencia de enfermedades que ya cuentan en este grupo profesional antes de la pandemia, los resultados de dicha sumatoria de factores producen enfermedades como depresión, ansiedad, problemas musculares y estrés laboral.

Al respecto, Choy y Wong (2017): definen al agotamiento emocional como una respuesta prolongada a los factores de estrés emocionales e interpersonales prolongados. En el escenario descrito anteriormente, los odontólogos de los centros de salud no tienen el tiempo ni la manera de recuperarse ante la exposición de las múltiples tareas durante la pandemia, razón por la cual son frecuentes las manifestaciones de cansancio, 
extenuación y debilidad. Esta realidad repercute en el ámbito laboral y ocasiona conflictos y desavenencias que constituyen un círculo perpetuo de estrés.

Por su parte, Jiménez-Ortiz, Islas-Valle, Jiménez-Ortiz, PérezLizárraga, Hernández-García y González-Salazar (2019): argumentan que el impacto en la salud mental incide en el sistema inmunológico y disminuye la capacidad de combatir las enfermedades y mantener un medio interno constante. En este escenario, es inevitable que el desequilibrio entre las demandas laborales y los recursos del trabajador produzcan afectaciones físicas como astenia, pérdida de apetito, problemas digestivos, trastornos de sueño, trastornos neuromusculares, trastornos cardiovasculares, entre otros.

El Burnout somatiza el estrés e incrementa la vulnerabilidad del trabajador frente a siniestros, ya que, al estar abatido emocional y físicamente, disminuye la capacidad de atención, de alerta y de vigilia, con lo cual la probabilidad de que se accidente aumenta ostensiblemente.

Acorde con esto, Yslado, Norabuena, Loli, Zarzosa, Padilla, Pinto y Rojas (2019a): señalan que el individuo que sufre de Burnout es quien se cierra y pasa en negación frente a cualquier situación que implique su entera dedicación y entusiasmo, que se acompaña de comportamientos paranoides, sentimientos de vacío, fracaso, agotamiento, inquietud, nerviosismo, inclusive se comportan agresivos con pacientes, presentan dificultades con los compañeros de trabajo, teniendo repercusión familiar, generando conflictos interpersonales, y por último, manifestaciones emocionales, tales como: distanciamiento, baja autoestima, comportamiento de alto riesgo e inclusive intentos autolíticos, lo cual representa una de las etapas más difíciles por las que pasa.

En este orden de ideas, Saliba, Saliba, dos Santos, Leal y Ísper (2012): explica que la función laboral y el grado de capacitación que se recibe para una determinada tarea implica un problema en cuestión, pues los 
profesionales que no tienen claro en qué consisten sus funciones, tienden a presentar alto nivel de estrés. Por otro lado, los factores sociodemográficos y de carácter personal repercuten en el desempeño laboral, pues este último al darse inadecuadamente, puede ser el resultado de un pobre balance en la relación hogar y trabajo, con severas implicaciones que convergen en pobreza, desempleo y condiciones de vida precarias.

Ante esto, Ferrel, Ferrel y Bracho (2018): sostienen que es evidente que las repercusiones no tienen una base sustancial en el desempeño profesional, y que el escenario muestra un notorio impacto social, pues el excesivo estrés en el personal genera un grave daño al sistema sanitario, aumenta los costos sociales y económicos, haciendo del Síndrome de Burnout un problema de importancia social y de salud pública.

Adicionalmente, Deeb, Braun, Carrico, Kinser, Laskin y Golob (2018): reflexionan sobre los desafíos de ser odontólogo por su alto riesgo de presentar estados afectivos negativos, que a largo plazo tienen consecuencias negativas por estrés en la práctica profesional siendo significativas al presentar agotamiento, despersonalización y baja eficacia profesional, lo cual repercute en el desempeño laboral.

Conforme a Mafla, et al. (2014): los profesionales de la salud bucal están sometidos a altas exigencias y desarrollan habilidades intelectuales, psicosociales e interpersonales, en busca de mecanismos de afrontamiento ante las diversas situaciones que se puedan generar en el campo laboral, tratando de ser altamente competitivos.

En atención a, García y Mendoza (2019a): en la actualidad se vienen dando una serie de modificaciones en el sistema de salud, donde el personal tienen que ajustarse a los requerimientos y constantes exigencias por parte de la institución a la que se deben, es así, que el panorama es alarmante y preocupante en cuanto a la presencia del Síndrome de Burnout y aún más en profesionales como los odontólogos, que constantemente se enfrentan a 
situaciones de desgaste psicoemocional, pues el análisis individualizado de cada paciente, la necesidad de brindar un servicio ideal, genera un clima de ansiedad, desequilibrio conductual, además el estar expuestos a diversos peligros, que sobreestima su capacidad para hacerle frente a sus funciones, genera inseguridad, pérdida del autoestima y deficiente resolución activa de problemas.

En correspondencia a las exigencias y situaciones constantes de demanda por parte de los pacientes, los cuales en ocasiones se muestran ansiosos y no colaboradores, se añade a esto los procedimientos complejos y extensos, carga horaria, comunicación poco efectiva con personal que labora, lo cual agrava la situación de eficacia, efectividad y adecuada relación odontólogo-paciente. Es importante mencionar ciertos factores que inciden en el aumento de la tensión de los odontólogos, estos son: realizar su trabajo en un área limitada, posturas forzadas, restringidas y prolongadas para obtener una mejor visibilidad exclusivamente a la cavidad bucal, ambiente de trabajo ruidoso debido a las piezas de mano, insuficiente iluminación y escasa ventilación. Por tanto, se ha visto necesario generar estrategias y programas para disminuir o prevenir el Síndrome de Burnout.

Además, en la actualidad laboran con una nueva modalidad ante la COVID-19, y están más expuestos que en otras carreras por los tratamientos que son a corta distancia entre paciente y profesional, incluyendo los trajes de medidas de seguridad que utilizan durante toda su jornada de trabajo, entre todo esto tiene más roles dentro del equipo operativo de salud, para maximizar el rendimiento y efectividad en el sistema de salud, lo cual ayuda a descongestionar el servicio y permite salvaguardar vidas.

El objetivo del estudio fue determinar el grado de afectación del Síndrome de Burnout en odontólogos del Seguro Social Campesino de Chimborazo en el año 2020. 


\section{Metodología}

Para la presente investigación, se consideró un estudio con diseño observacional, transversal, prospectivo y descriptivo. La población de estudio estuvo conformada por 43 odontólogos del Seguro Social Campesino de la Provincia de Chimborazo, no siendo necesario calcular una muestra, por lo que se seleccionó a la totalidad de dicha población utilizando como criterios de inclusión tener disposición para participar en el estudio y trabajar en la institución, mientras que como criterios de exclusión se tuvo en cuenta aquellos odontólogos que se encontraran hospitalizados en el momento de desarrollarse el estudio, no existiendo ningún caso en este sentido.

Se aplicó una encuesta a través del test de Maslach Burnout InventoryGeneral Survey (MBI-GS). A la encuesta de agotamiento emocional, se le añadieron preguntas nominales como el género; edad; estado civil; antigüedad; y tipo de jornada laboral. Posteriormente, se calcularon los baremos de la escala del instrumento para la obtención de los resultados con los que se pudo confirmar el análisis estadístico descriptivo. Se empleó la prueba estadística de Chi-cuadrado $\left(\mathrm{X}^{2}\right)$ para confirmar si las variables categóricas nominales presentaban significancia estadística ( $p$-valor $<=0.05$ ) con el grado de exposición de los tres factores de la encuesta: despersonalización, agotamiento emocional y eficacia profesional.

Para Sánchez, Vega, Gómez y Vilema (2020): sirvió de referencia para interpretar el Odds ratio (OR) en base al estudio de la asociación entre variables sociodemográficas y el Síndrome de Burnout. Este estadístico fue utilizado para confirmar la probabilidad de ocurrencia de los factores del burnout con relación a la presencia de las variables categóricas nominales.

Los métodos y procedimientos desarrollados se aprobaron por el Comité de Bioética de la Universidad Regional Autónoma de los Andes (UNIANDES), Ecuador, alineándose con la Declaración de Helsinki del año 1975 y sus posteriores actualizaciones. Además, se requirió la autorización por 
parte de la dirección de la institución objeto de estudio, que simultáneamente con el acuerdo de confidencialidad con cada trabajador participante en la investigación, fue considerado como consentimiento informado para realización del estudio.

\section{Resultados}

Respecto a las variables nominales, se evidenció que dentro de la población de estudio 29 pertenecían al género femenino $(67,4 \%)$ y 14 al género masculino $(32,6 \%)$.

Dentro de la variable edad, predominó el grupo etario comprendido entre 30 y 39 años ( $N=24$ y 55.8\%); seguido por el grupo de 20 a 29 años ( $N=8$ y 18,6\%); grupo de 40 a 49 años ( $N=6$ y 14,0\%); y finalmente el grupo de 50 a 59 años ( $N=5$ y 11,6\%); mientras que respecto a la variable estado civil, 24 eran casados (55,8\%); 17 eran solteros (39,5\%); y 2 eran divorciados $(4,7 \%)$.

La variable antigüedad laboral evidenció un predominio del período comprendido entre 1 y 5 años ( $N=21$ y 48,8\%); seguido por el período enmarcado entre 5 y 15 años ( $N=17$ y 39,5\%); período menor de seis meses ( $N=3$ y $7,0 \%)$; y finalmente el período mayor de 15 años ( $N=2$ y $4,7 \%)$.

Finalmente, en la variable relacionada con el tipo de jornada laboral predominó la jornada completa ( $\mathrm{N}=40$ y $93,0 \%)$, superior claramente a la jornada parcial $(\mathrm{N}=3$ y $7,0 \%)$.

En esencia se evidencia dentro del perfil sociodemográfico que el género femenino fue el que destacó con mayor frecuencia, al igual que el estado civil casado, el rango de edad con mayor frecuencia corresponde a adultos jóvenes, con una antigüedad laboral menor a 5 años y jornada de trabajo completa.

A continuación, se exponen las frecuencias y porcentajes relacionados con el Burnout, según sus dimensiones. De los resultados del Test MBI-GS se obtuvo que existe presencia de las tres dimensiones del Burnout en los 
profesionales odontólogos, no obstante, la eficacia profesional (9,3\% Muy Alto y $7,0 \%$ Alto) y el desgaste emocional (14,0\% Alto) predominan en relación al cinismo, que solo tuvo $2,3 \%$ Alto y $4,7 \%$ Alto.

Se aprecia en la tabla 1, una minoría de odontólogos que mostraron desgaste emocional, 6 de ellos valorados en alto (13,9\%), 2 considerados como medio alto $(4,7 \%)$ y 11 considerados como medio bajo $(25,6 \%)$, mientras que el resto no evidenció tener desgaste emocional, al ser valorados como bajo (11) o muy bajo (13).

Igualmente, una minoría de odontólogos mostraron cinismo, 1 de ellos definido como muy alto; 2 definidos como alto; 5 clasificados como medio alto y 9 definidos como medio bajo. Los otros no evidenciaron cinismo, siendo valorados 6 de ellos como bajo y 20 como muy bajo.

La eficacia profesional se evidenció igualmente en una minoría de odontólogos, desglosándose en 4 considerados en muy alto; 3 valorados de alto; 2 considerados medio alto y 1 clasificado medio bajo, mientras que la mayoría no evidenció eficacia profesional, pues 7 de ellos fueron clasificados bajo y 26 muy bajo.

Tabla 1. Frecuencia y porcentaje de Burnout según sus dimensiones.

\begin{tabular}{|c|c|c|c|c|c|c|c|c|c|c|c|c|}
\hline \multirow{2}{*}{ DIMENSIONES } & \multicolumn{2}{|c|}{ Muy Alto } & \multicolumn{2}{|c|}{ Alto } & \multicolumn{2}{|c|}{ Medio Alto } & \multicolumn{2}{|c|}{ Medio Bajo } & \multicolumn{2}{|c|}{ Bajo } & \multicolumn{2}{|c|}{ Muy Bajo } \\
\hline & FR & $\%$ & FR & $\%$ & FR & $\%$ & FR & $\%$ & FR & $\%$ & FR & $\%$ \\
\hline Desgaste emocional & 0,0 & 0,0 & 6 & 13,9 & 2 & 4,7 & 11 & 25,6 & 11 & 25,6 & 13 & 30,2 \\
\hline Cinismo & 1 & 2,3 & 2 & 4,7 & 5 & 11,6 & 9 & 20,9 & 6 & 14,0 & 20 & 46,5 \\
\hline Eficacia profesional & 4 & 9,3 & 3 & 7,0 & 2 & 4,6 & 1 & 2,3 & 7 & 16,3 & 26 & 60,5 \\
\hline
\end{tabular}

Fuente: Los Autores (2020).

En la tabla 2 se exponen los resultados de la prueba de Chi-cuadrado entre las variables nominales y el Burnout, resaltándose con un "*” los tres valores cuya significancia estadística tuvo un $p$-valor $<=$ de 0,05.

En dicha tabla se observa que con respecto a la antigüedad laboral se evidenció que está relacionada de forma estadísticamente significativa con la 
eficacia profesional $(0,005)$, mientras que respecto a la jornada de trabajo se demostró una relación estadísticamente significativa con el cinismo $(0,045)$ y con la eficacia profesional $(0,005)$.

Tabla 2. Significancia estadística (p-valor) en la prueba de Chi-cuadrado ( ${ }^{*}$ Valores inferiores al p-valor de 0,05).

\begin{tabular}{|c|c|c|c|}
\hline VARIABLES SOCIO DEMOGRÁFICAS & $\begin{array}{c}\text { Desgaste } \\
\text { Emocional }\end{array}$ & Cinismo & $\begin{array}{c}\text { Eficacia } \\
\text { Profesional }\end{array}$ \\
\hline Género & 0,916 & 0,166 & 0,649 \\
\hline Edad & 0,475 & 0,331 & 0,493 \\
\hline Estado civil & 0,754 & 0,691 & 0,755 \\
\hline Antigüedad laboral & 0,089 & 0,088 & $0,005^{*}$ \\
\hline Jornada de trabajo & 0,395 & $0,045^{*}$ & $0,005^{*}$ \\
\hline & $\mathbf{N}=\mathbf{4 3}$ \\
\hline
\end{tabular}

Fuente: Los Autores (2020).

En cuanto a la frecuencia (OR) respecto a presentar o no Burnout, en el caso de la eficacia profesional 33 profesionales $(76,7 \%)$ no presentaron y $10(23,3 \%)$ si presentaron. Respecto al desgaste emocional 24 no presentaron $(55,8 \%)$ y 19 si presentaron (44,2\%), mientras que, con relación al cinismo, 26 no presentaron $(60,5 \%)$ y 17 si presentaron $(39,5 \%)$.

De los 43 profesionales, dos $(4,7 \%)$ presentaron las tres dimensiones de Burnout, 14 (32,6\%) presentaron dos dimensiones, 12 (27,9\%) presentaron una sola dimensión y 15 (34,9\%) no tuvieron ninguna.

\section{Discusión}

De acuerdo con Lara (2020): sobre la prevalencia del Síndrome de Burnout en estudiantes que cursan clínica en la facultad de Odontología de la Universidad de las Américas UDLA (Ecuador), donde se trabaja con una muestra similar a la de la presente investigación de 69 personas, se obtiene que $53,6 \%$ presenta desgaste emocional, 46,4\% cinismo, que es compatible con el actual estudio donde se obtiene un 44,2\% (considerando 19 casos de un total de 43, sumando 6 valorados de alto, 2 valorados de medio y 11 
valorados de medio bajo) de desgaste emocional, 39,5\% de cinismo y en relación con la eficacia profesional se obtiene mayor eficacia profesional en el estudio antes mencionado. Finalmente, respecto al Burnout se obtiene 14,5\%, no obstante, en el presente estudio se presenta en un porcentaje más bajo de un $4,7 \%$.

Con respecto al presente estudio, la baja eficacia profesional influye negativamente en la vida laboral, la misma que comprende a 33 profesionales $(76,7 \%)$, seguido del desgaste emocional que afecta a $19(44,1 \%)$, el cinismo con 17 odontólogos (39,5\%), donde 16 (37,2\%) de ellos presentan de dos a tres dimensiones, siendo diferente al estudio que realiza Ogdon y DíazReissner (2017b): sobre el Síndrome de Burnout en odontólogos de la XVIII Región Sanitaria del Ministerio de Salud Pública y Bienestar Social de Paraguay, en el cual se incluye a 83 odontólogos y se encuentran bajos valores de cansancio emocional.

Según el artículo de Preciado, Pozos-Radillo, Colunga-Rodríguez, Vázquez-Goñi y Ávalos-Latorre (2017): sobre relación entre factores psicosociales, agotamiento emocional laboral y Burnout en odontólogos mexicanos, realizado a 72 docentes dentistas del sector público y 72 de la práctica privada, se encuentra que el $25 \%$ de los profesionales de la práctica pública, y el $22 \%$ de la práctica privada presentan cansancio emocional, se registran niveles de cinismo de $26 \%$ en la práctica pública y $28 \%$ en la práctica privada, se relaciona con el presente estudio, en donde se encuentra desgaste emocional en $44,2 \%$ y cinismo en $39,5 \%$, y difiere en relación a la eficacia profesional, donde se presenta en $8 \%$ de personal de la práctica pública y 15 $\%$ de la práctica privada, realizando una comparación con los participantes del área publica se evidencia que no tiene relación con la presente investigación, donde el $44,2 \%$ presenta desgaste emocional, cinismo con 39,5\% y difiere en la eficacia profesional con $76,8 \%$.

En el estudio sobre la correlación entre inteligencia emocional y el 
Síndrome de Burnout que se realizó a 90 odontólogos del Ministerio de Salud de Puno, para Ecuador García y Mendoza (2019b): se presenta de la manera siguiente: $86,7 \%$ de profesionales con baja despersonalización, 61,1\% con bajo agotamiento personal; en cambio, el 67,8\% presenta alta realización personal. Estos resultados no se relacionan con los datos que se obtienen en el presente estudio, donde la baja eficacia profesional se encuentra en un porcentaje más alto $76,8 \%$ seguido del desgaste emocional con 44,2\% y el cinismo $39,5 \%$, estos dos últimos en menor porcentaje en relación con el estudio mencionado.

En esa misma línea, Yslado, et al. (2019b), realizaron una investigación en dos hospitales de Perú, sobre el Síndrome de Burnout y la satisfacción laboral en profesionales de la salud que se desarrolló con 177 profesionales de la salud, la tasa de prevalencia del Burnout es de 33,3\% y existe relación entre el Burnout y la satisfacción laboral, donde la dimensión que predomina es el cinismo en $37,2 \%$, lo cual no se relaciona con el presente estudio, puesto que se encuentra Burnout en un porcentaje más bajo en un 4,7\% y se relaciona debido a que el porcentaje que se obtiene de cinismo fue similar en un $39,5 \%$.

Interpretando a Mera (2017): el Síndrome de Burnout en odontólogos de los distritos de salud de la Provincia de Bolívar, evidenció la presencia de Burnout como media; Además, un nivel de agotamiento emocional medio en $41(42,7 \%)$, el nivel de despersonalización bajo en 66 (68,8\%), realización personal bajo en $53(55,2 \%)$, lo cual influye en su vida personal y laboral en la su población. Por su parte los resultados de la presente investigación reflejan que la prevalencia del Síndrome de Burnout en los odontólogos del Seguro Social Campesino de Chimborazo es baja, donde predomina, además, la baja eficacia profesional la misma que comprende 33 profesionales $(76 \%)$, seguido del desgaste emocional que afecta a 19 (44,2\%) profesionales de odontología, y el cinismo con 17 (39,5\%).

En referencia al tema, Pinheiro, Burges y Remor (2020): realizan un 
análisis bajo el estrés de trabajo y Burnout entre higienistas dentales, que incluye a 348 profesionales de las ocho gerencias de salud del distrito en la ciudad de Porto Alegre, Brasil, en los resultados del estudio se indica la importancia de la empatía y la relación con el agotamiento emocional, además, la angustia personal es un factor de riesgo, mientras que en la presente investigación la antigüedad laboral está relacionada con la eficacia profesional y la jornada de trabajo con el cinismo y baja eficacia en la ejecución de las laborales.

En un estudio que realizado por Myoung-Uk, Seong-Hwa, Eun-Kyong, Youn-Hee y Keun-Bae (2015): sobre Burnout y sus factores en dentistas coreanos, en el cual se realizó en una encuesta mediante el cuestionario Maslach Burnout Inventory a 1000 dentistas, se obtuvo como resultados que las puntuaciones de agotamiento emocional, despersonalización y bajo sentido de logro personal, son altas con 41,2\%, 55,9\%, y 41,4\% respectivamente y es más probable que el agotamiento alto se produzca por relación a alguna variable sociodemográfica relacionada con el trabajo, mientras que en el presente estudio predomina la baja eficacia profesional con $76,7 \%$, agotamiento emocional con $44,2 \%$ y cinismo con $39,5 \%$.

En el escrito de Huria, Bağiş, Eren, Başibüyük, Şahin, Umaroğlu y Orhan (2016): sobre Burnout y participación ocupacional entre odontólogos con responsabilidades docentes en universidades, se obtiene que el $8 \%$ de los dentistas presentan agotamiento emocional significativo, 11\% presentan despersonalización y agotamiento emocional, el 17\% poseen baja realización personal y agotamiento emocional, 36\% alto nivel de agotamiento emocional, con respecto al presente estudio donde se evidencia la baja eficacia profesional la misma que comprende (76\%), donde el desgaste emocional afecta un $44,2 \%$ profesionales y el cinismo con $39,5 \%$ en profesionales de la odontología. 


\section{Conclusiones}

En los Odontólogos del Seguro Social Campesino de Chimborazo, Ecuador, se encontró un bajo porcentaje Síndrome de Burnout que aplica a dos profesionales, catorce odontólogos presentan las dos dimensiones, doce profesionales presentaron una dimensión y quince profesionales no presentan rasgos relacionados con el Burnout.

Se puede inferir que la antigüedad laboral está relacionada con el cinismo y la eficacia profesional, no obstante, esta última está determinada también por la jornada de trabajo.

Las tres dimensiones se encontraron presentes en los profesionales odontólogos, la eficacia profesional se localizó mayormente afectada, seguido del cansancio emocional y el cinismo.

A partir de lo cual nos permite tomar acciones y establecer lineamientos para controlar las dimensiones afectadas y evitar la incidencia del Síndrome de Burnout siendo necesario diseñar e implementar estrategias mediante un programa integral psicosocial en el Seguro Social Campesino de Chimborazo, Ecuador, que incluya talleres, charlas, seminarios con temas de realización personal y profesional, para mejorar los factores que incitan el Burnout esto permitirá mejorar el desempeño laboral y por ende sus emociones, a fin de que los profesionales odontólogos tengan un ambiente de trabajo adecuado y agradable para que puedan realizar su trabajo con calidad, eficiencia y satisfacción personal.

\section{Referencias}

Chacaltana, K., \& Rojas, L. (2019). Persistencia del síndrome burnout en internos de medicina en hospitales de Lima, Perú (año 2018). Investigación en Educación Médica, 8(32), 9-15, e-ISSN: 2007-865X. Recuperado de: https://www.redalyc.org/articulo.oa?id=349763025002 Choy, HB., \& Wong, M. (2017). Occupational stress and burnout among 
OAI-PMH: http://www.indteca.com/ojs/index.php/Revista Scientific/oai

Artículo Original / Original Article

Hong Kong dentists. Hong Kong Medical Journal, 23(5), 480-488.

Recovered from: https://doi.org/10.12809/hkmj166143

Deeb, G., Braun, S., Carrico, C., Kinser, P., Laskin, D., \& Golob, J. (2018).

Burnout, depression and suicidal ideation in dental and dental

hygiene students. European Journal of Dental Education, 22(1), e70e74, e-ISSN: 1396-5883. Recovered from:

https://doi.org/10.1111/eje.12259

Ferrel, L., Ferrel, F., \& Bracho, K. (2018). Impacto del Síndrome de Burnout Académico en el Bajo Rendimiento y la Salud Mental en Estudiantes Universitarios. Conocimiento, Investigación y Educación, 2(6), 45-53, e-ISSN: 2539-5289. Colombia: Universidad de Pamplona.

García, B., \& Mendoza, J. (2019a,b). La correlación entre inteligencia emocional y síndrome de Burnout. Evidencias en Odontología Clínica, 5(2), 47-59, e-ISSN: 2521-2087. Recuperado de: https://revistas.uancv.edu.pe/index.php/EOC/article/view/787

Huayhua, K. (2017). Influencia del Síndrome de Burnout en el Rendimiento Académico de los Estudiantes de la Clínica Odontológica. Revista Científica Investigación Andina, 17(1), 245-253, e-ISSN: 1994-8077. Recuperado de:

https://revistas.uancv.edu.pe/index.php/RCIA/article/view/329

Huria, M., Bağiş, N., Eren, H., Başibüyük, O., Şahin, S., Umaroğlu, M., \& Orhan, K. (2016). Burnout and occupational participation among dentists with teaching responsibilities in universities. Psychology, Health \& Medicine, 22(6), 693-700, e-ISSN: 1354-8506. Recovered from: https://doi.org/10.1080/13548506.2016.1210177

Jiménez-Ortiz, J., Islas-Valle, R., Jiménez-Ortiz, J., Pérez-Lizárraga, E., Hernández-García, M. \& González-Salazar, F. (2019). Emotional exhaustion, burnout, and perceived stress in dental students. Journal of International Medical Research, 47(9), 4251-4259. 
Recovered from: https://doi.org/10.1177/0300060519859145

Lara, N. (2020). Prevalencia del síndrome de Burnout en estudiantes que se encuentran cursando la clínica $V$ en la facultad de odontología de la Universidad de las Américas en el periodo 2020-1. Trabajo de Titulación. Quito, Ecuador: Universidad de las Américas. Recuperado de: http://dspace.udla.edu.ec/handle/33000/11960

Mafla, A., Villa-Torres, L., Polychronopoulou, A., Polanco, H., Moreno-Juvinao, V., Parra-Galvis, D., ... Divaris, K. (2014). Burnout prevalence and correlates amongst Colombian dental students: the STRESSCODE study. European Journal of Dental Education, 19(4), 242-250, e-ISSN: 1396-5883. Recovered from: https://doi.org/10.1111/eje.12128

Mera, E. (2017). Prevalencia del Síndrome de Burnout en odontólogos de los Distritos de Salud de la provincia de Bolívar. Quito, Ecuador: Universidad Central del Ecuador. Recuperado de:

http://www.dspace.uce.edu.ec/handle/25000/13187

Meyerson, J., Gelkopf, M., Eli, I., \& Uziel, N. (2020). Burnout and professional quality of life among Israeli dentists: the role of sensory processing sensitivity. International Dental Journal, 70(1), 29-37, e-ISSN: 0020-6539. Recovered from:

https://doi.org/10.1111/idj.12523

Myoung-Uk, J., Seong-Hwa, J., Eun-Kyong, K., Youn-Hee, C., \& Keun-Bae, S. (2015). Burnout and its related factors in Korean dentists. International Dental Journal, 65(1), 22-31, e-ISSN: 0020-6539. Recovered from: https://doi.org/10.1111/idj.12142

Ogdon, MA., \& Díaz-Reissner, CV. (2017a,b). Síndrome de Burnout en odontólogos de la XVIII Región Sanitaria del Ministerio de Salud Pública y Bienestar Social, Paraguay. Revista de salud pública del Paraguay, 7(1), 21-27, e-ISSN: 2307-3349. Recuperado de: 
http://scielo.iics.una.py/scielo.php?script=sci abstract\&pid=S2307$\underline{33492017000100021}$

Pinheiro, J., Burges, J., \& Remor, E. (2020). Associação da empatia e do estresse ocupacional com o burnout em profissionais da atenção primária à saúde. Ciência \& Saúde Coletiva, 25(9), 3635-3646. Recuperado de: https://doi.org/10.1590/1413-81232020259.30672018

Preciado, M., Pozos-Radillo, B., Colunga-Rodríguez, C., Vázquez-Goñi, J., \& Ávalos-Latorre, M. (2017). Relación entre factores psicosociales, agotamiento emocional laboral y burnout en odontólogos mexicanos. Universitas Psychologica, 16(2), 1-11, e-ISSN: 1657-9267. Recuperado de: https://doi.org/10.11144/Javeriana.upsy16-2.refp

Saliba, C., Saliba, O., dos Santos, R., Leal, R., \& Ísper, A. (2012). Burnout en estudiantes de Odontología: Evaluación a través MBI - versión estudiantes. Medicina y Seguridad del Trabajo, 58(229), 327-334, eISSN: 1989-7790. Recuperado de:

https://dialnet.unirioja.es/servlet/articulo?codigo $=5382306$

Sánchez, B., Vega, V., Gómez, N., \& Vilema, G. (2020). Estudio de casos y controles sobre factores de riesgo de diabetes mellitus tipo 2 en adultos mayores. Universidad y Sociedad, 12(4), 156-164, e-ISSN: 2218-3620. Recuperado de:

https://rus.ucf.edu.cu/index.php/rus/article/view/1626

Yslado, R., Norabuena, R., Loli, T., Zarzosa, E., Padilla, L., Pinto, I., Rojas, A. (2019a,b). Síndrome de burnout y la satisfacción laboral en profesionales de la salud. Horizonte Médico, 19(4), 41-49, e-ISSN: 2542-2987. Recuperado de:

https://doi.org/10.24265/horizmed.2019.v19n4.06 


\section{Caterine Nataly Orozco Orozco}

e-mail: kathi2815@hotmail.com

Nacida en Riobamba, Ecuador, el 2 de agosto del año

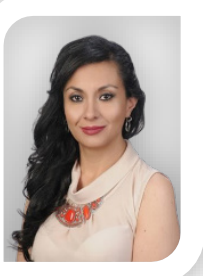
1988. Odontóloga de la Universidad Nacional de Chimborazo (UNACH); Odontóloga tratante del Seguro Social Campesino (IESS); trabajo basado en prevención, atención y curación, de grupos prioritarios en el área rural, enfocándome a que el paciente tenga un buen estado de Salud bucal y en General; Ejerzo en consulta privada realizando una práctica de atención integral, fomento y prevención con niños, adolescentes, adultos y embarazadas; he participado en diferentes Congresos, Seminarios y Talleres dentro del área de la Odontología, la Educación y la Investigación. 


\section{Darwin Raúl Noroña Salcedo}

e-mail: darwin norona@yahoo.com

Nacido en Quito, Ecuador, 13 de marzo del año 1983.

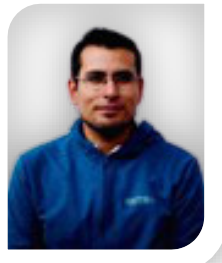

Docente de la Maestría de Salud Ocupacional en el módulo de Factores Psicosociales de Riesgo a la Salud de la Universidad Regional Autónoma de los Andes (Uniandes); Docente Titular en el Instituto Superior Tecnológico Sucre, impartiendo las asignaturas de Estadística, Metodología de Investigación, Riesgos, Emergencias y Psicología del Consumidor; Profesional en el área de estadística, cambio de cultura organizacional, violencia de género, discriminación e interculturalidad, y en investigación en seguridad y salud ocupacional; con aptitudes de liderazgo, investigación, desarrollo y ejecución de proyectos técnicos; especial énfasis en dirección de equipos de trabajo, consultorías profesionales y en docencia universitaria. 


\section{Vladimir Vega Falcón}

e-mail: vega.vladimir@gmail.com

Nacido en Matanzas, Cuba, el 18 de agosto del año

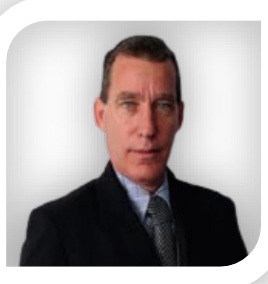
1964. PhD en Ciencias Económicas; Categoría Docente Principal de Profesor Titular (Catedrático); actualmente Docente Investigador con dedicación a Tiempo Completo, Titular Principal Nivel de Escalafón 3 de la Universidad Regional Autónoma de los Andes (UNIANDES), Ecuador; he colaborado sistemáticamente como docente de pregrado y postgrado en universidades de varios países, abordando diversas temáticas, entre las que se encuentran: "Contabilidad de Costos", "Contabilidad de Gestión Avanzada", "Planeación Estratégica y Cuadro de Mando Integral", "Herramientas gerenciales para toma de decisiones", "Capital Intelectual", "Gestión Turística", "Metodología de la Investigación", "Redacción de artículos científicos".

El contenido de este manuscrito se difunde bajo una Licencia de Creative Commons ReconocimientoNoComercial-Compartirlgual 4.0 Internacional 